\title{
Screening Infant Mental Health Indicators
}

\section{An Early Head Start Initiative}

\author{
Katbleen M. Baggett, PhD; Leslie Warlen, MPH; \\ Jenny L. Hamilton, MA; Jennifer L. Roberts, MC; \\ Martba Staker, MA, MS
}

\begin{abstract}
There is growing recognition of the multiple and complex needs of families who request services from early head start (EHS) programs. One of the challenges of EHS programs is to screen multiple risks more efficiently so that families can be referred for appropriate support services and so that families who are most in need of EHS services are able to receive them. Meeting this challenge has been cited as a priority for EHS programs and is central to better understanding and addressing infant mental health needs among families who come into contact with EHS programs. Community, state, and federal monitoring systems have been identified as an important mechanism for tracking and improving the well-being of America's children and adolescents. To the extent that predictors of infant mental health problems are known, communities can develop monitoring systems for the purposes of prevention and treatment. The purpose of this article is to identify common limitations of screening and referral approaches in EHS, to describe the process by which one EHS program has begun to address such limitations, and to highlight indications of system effectiveness as well as plans for future evaluation. Key words: community collaboration, early bead start, infant mental bealth indicators, screening/referral systems
\end{abstract}

$\mathbf{I}^{\mathbf{r}}$ NFANT MENTAL HEALTH has been defined as "the state of emotional and social competence in young children who are developing within the interrelated contexts of biology, relationship, and culture" (Zeanah \& Zeanah, 2000, p. 21). Social-emotional com petence has been defined as "children's reac-

Autbor Affiliations: University of Kansas (Dr Baggett), University of Kansas Medical School (Mss Warlen, Hamilton, Roberts and Staker), Kansas City.

Preparation of this manuscript was supported by the Healthy Tomorrows Partnership for Children Program Grant 1 H17MC02507-01-00 from the American Academy of Pediatrics (AAP) and the Maternal and Child Health Bureau (MCHB). The opinions expressed berein do not necessarily reflect the position or policy of $A A P$ or $M C H B$, and no official endorsement should be inferred.

Corresponding Autbor: Katbleen M. Baggett, PbD, 650 Minnesota Ave., 2nd Floor, Kansas City, Kansas 66101 (kbaggett@ku.edu). tions and responses that produce satisfying interactions to the individual and his or her social world" (Squires \& Bricker, 2007, p. 10). While infant mental health theory emphasizes the importance of understanding child, family, and community contexts in the development and expression of infant mental health competencies and problems, there is a striking gap between infant mental health theory and operational mechanisms for screening indicators of infant mental health within communities. Screening systems are needed to identify existing social-emotional concerns in very young children as well as to identify significant child, parent, and family risks that can contribute to the development of early social-emotional problems (Appleyard, Egeland, van Dulmen, \& Sroufe, 2005; Mrazek, Biglan, \& Hawekins, 2004). This is especially the case for programs, such as early head start (EHS), which are charged with the task of supporting early social-emotional development by responding to social-emotional delays and 
difficulties before they become debilitating and by providing appropriate referrals to address a wide array of risk conditions. The purposes of this article are to (1) identify common limitations of and challenges to screening and referral approaches within EHS, (2) highlight considerations for addressing such challenges, and (3) provide an illustration by describing how one EHS program has begun to address these challenges.

\section{COMMON LIMITATIONS OF SCREENING AND REFERRAL APPROACHES}

A variety of factors limit the effectiveness and efficiency of screening and referral approaches within EHS as well as within other early intervention programs (eg, those for children with or at-risk for developmental disabilities). These factors include (1) narrow eligibility screening, (2) significant delays and interruptions in screening and referral due to long waiting lists, (3) unsystematic approaches to screening and referral, and (4) lack of evaluation efforts to inform and guide more effective screening and referral. While these factors are relevant to effectiveness of screening and referral processes in general, they are paramount for infant mental health intervention that requires response within the relatively brief window of infancy (Bricker, Davis, \& Squires, 2001; Squires \& Bricker, 2007).

First, narrow screening is commonly done to determine eligibility status for a particular program (see Harbin, 2005). Examples include (1) a Part C program, which fails to screen for and refer social-emotional problems or high-risk parent-child interactions and (2) an EHS Program that fails to screen and refer for child cognitive and communication delays. In each of these cases, opportunities for early detection are lost because of narrow agency screening that failed to detect concerns, which if identified and referred, would have resulted in eligibility for other relevant early intervention services. When single agencies conduct narrow screening and fail to establish strong interagency collaboration, op- portunities to provide appropriate referrals are lost (see Harbin, 2005).

Second, opportunities for timely and appropriate referrals are often lost because of significant delays between the time families first seek EHS services and the time that they actually receive them. Current estimates indicate that EHS serves only $2 \%$ of the eligible population in the nation (The Child Mental Health Foundations and Agencies Network, 2000). Many programs can serve only a fraction of families who are eligible for services. Consequently, long wait lists can result in significant service delays in which a parent may seek EHS services during pregnancy but not receive any screening or referral services for months, years, or not at all. Such lapses can occur because of a family remaining on a waiting for so long that the child is much older at the time an EHS opening finally does occur, and, in some cases, the child may have actually become too old to meet eligibility for EHS enrollment. Opportunities for screening and referral are also lost when programs are unable to locate families after a long period on a waiting list.

Third, approaches to referral are often unsystematic and lack either formal screening or the use of established measures with demonstrated detection sensitivity. This limitation is especially problematic when referrals are needed to address concerns that may fall outside the scope of EHS program expertise (eg, maternal depression, maternal substance abuse, child social emotional concerns, etc). To improve screening effectiveness, EHS programs need screening measures that are brief, sensitive, reliable, and inexpensive (see Gilliam, Meisels, \& Mayes, 2005; Glascoe, Foster, \& Woolraich, 1997; Windham et al., 2004).

Finally, screening and referral systems seldom include an evaluation component to inform and guide screening and referral practices. Better understanding of families' engagement in the screening and referral process, as well as their satisfaction with that process, are essential for developing more effective and efficient systems (Harbin, 2005). 
Lack of such information impedes community stakeholders in understanding and responding in meaningful ways to gaps between family needs and community services.

\section{CONSIDERATIONS FOR ADDRESSING LIMITATIONS AND CHALLENGES}

Integrated point of access (POA) models provide an innovative and useful conceptual framework (see Harbin, 2005) for addressing common barriers described above. POA refers to the point at which concerns about development or risks to development are examined in a manner that permits children and their families to be linked to a full array of resources and services within a community (Harbin, 2005). Exemplary POA models reflect integration of diverse disciplines and agencies for the purpose of carrying out child-find activities. In describing important considerations for designing POA systems relevant to common limitations, 6 system-level outcome goals have been identified by diverse stakeholders (see Harbin 2005; Harbin et al., 2004). These goals include (1) identification of concerns as early as possible, (2) provision of easy access for diverse families, (3) timely entry and rapid delivery of screening and referral services, (4) provision of full access to a wide array of resources, (5) promotion of consumer knowledge for navigating service delivery systems to access services, and (6) promotion of high family satisfaction.

\section{ONE EHS PROGRAM'S RESPONSE: CONNECTIONS}

Connections is a POA screening and referral system initiated by an EHS program in a Midwestern metropolitan area in collaboration with a wide array of child and family service agencies and a research partner. The purpose of Connections is to provide broad, efficient, and sensitive screening of infant mental health indicators among families for the express purpose of providing families with timely, appropriate referral support at the time they seek EHS services or the time they are referred directly for screening by other community agencies. Connections grew out of a series of community planning meetings designed to build community collaboration to promote the well-being of children, from birth to 5 years of age, and their families.

\section{Background}

In early 2000, approximately 60 community agencies began a collaboration that grew into the Children's Campus of Wyandotte County (CCWC). A driving force behind this collaboration was recognition of the need for a community-wide effort to promote school readiness by enhancing the well-being of children, from birth to 5 years of age, and their families. The purpose of the CCWC is to provide an integrated, seamless system of services for children birth to 5 years of age and their families. A variety of community challenges underscored the need for a high degree of interagency collaboration. In Wyandotte County, an inner-urban community affected by multiple stressors, $26.4 \%$ of children younger than 5 live in poverty (U.S. Census Bureau, 2000). Approximately half of all births in Wyandotte County occur to women living in the northeast corner of Wyandotte County (U.S. Census Bureau, 2000). Within these zip codes, 57\% of families earn incomes below the federal poverty guidelines, $34 \%$ of babies are born to mothers with no high school degree (Kansas Action for Children 2003), and 28\% represent births to adolescent mothers (Kansas Department of Health and Environment, 1999). Exposure to community violence profoundly affects the lives of families and children in this county. Approximately $27 \%$ of middle school and high school students living in Wyandotte County report that they do not feel safe in their home or community and $25.7 \%$ have been exposed to violent crime or drug selling in the neighborhood (Kansas Action for Children, 2003).

The most unique aspect of the CCWC is an extraordinary level of interagency collaboration for sharing human and financial resources to address the community challenges 
described above. While the CCWC provided and continues to provide a forum for building community collaboration, there was clear recognition that the CCWC needed a specific mechanism to generate timely, appropriate referrals across CCWC agencies spanning the domains of early education and intervention, family support, and health. Connections was the response to the CCWC's recognition of the need for such a screening and referral system. As one of the initiators and future residents of the CCWC, Project EAGLE Community Services spearheaded the first phase of development of Connections. In 1995, when the Administration for Children, Youth, and Families established EHS in response to the Head Start reauthorization, Project EAGLE became one of the first EHS programs and was selected to be one of the 17 research sites for the National EHS study. Project EAGLE is now the umbrella name for multiple communitybased programs, including EHS, Healthy Start, and a teen parenting support program.

\section{Unique features of Connections}

Consistent with the goals of exemplary POA models (see Harbin, 2005), the design and development of Connections involved several unique features including (1) commitment to strong interagency collaboration at every phase of development; (2) timely and appropriate referral support for all parents who are pregnant, who have a child younger than 1 year, and who seek EHS services in the county or who are referred specifically for screening by other collaborating agencies; and (3) application of a system evaluation component to inform ongoing system development as well as other community planning efforts. Development of Connections and its unique features, as described below, were designed specifically to address community challenges as well as common limitations of EHS referral systems.

\section{Strong interagency collaboration}

The Connections program was developed and continues to function through strong interagency collaboration efforts. Initially, rep-
Table 1. Types of collaborating agencies with established interagency agreements early intervention (Part C and Part B)

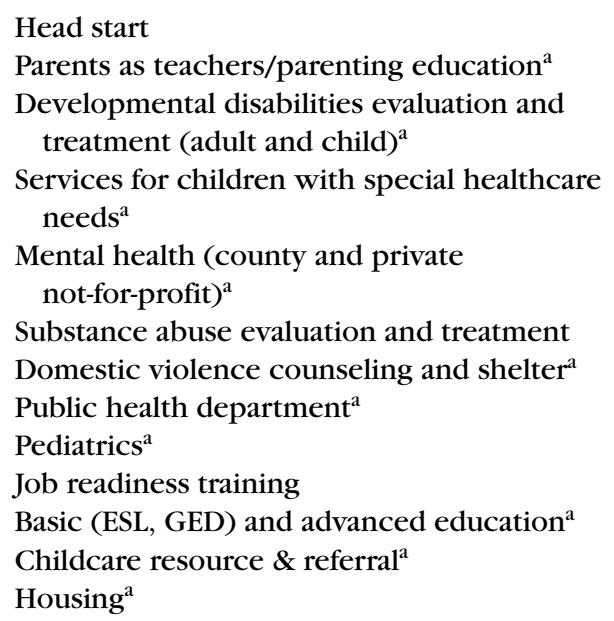

Head start

Parents as teachers/parenting education ${ }^{a}$

Developmental disabilities evaluation and treatment (adult and child) ${ }^{\mathrm{a}}$

Services for children with special healthcare needs ${ }^{\mathrm{a}}$

Mental health (county and private not-for-profit) ${ }^{\mathrm{a}}$

Substance abuse evaluation and treatment Domestic violence counseling and shelter ${ }^{\mathrm{a}}$ Public health department ${ }^{\mathrm{a}}$

Pediatrics $^{\mathrm{a}}$

Job readiness training

Basic (ESL, GED) and advanced education ${ }^{\mathrm{a}}$

Childcare resource $\&$ referral $^{\mathrm{a}}$

Housing ${ }^{\mathrm{a}}$

${ }^{\mathrm{a}}$ Types of Agencies Active on the Advisory Board

resentatives from various community agencies, who serve families with young children, were brought together to discuss the opportunity to collaborate on the development of Connections. By signing interagency agreements, 12 agencies formalized their partnership with Connections, and 7 additional agencies were also active partners during the early stages of development (Table 1). Interagency agreements were established to achieve the following tasks: map eligibility criteria across agencies and identify current overlaps and gaps in infant mental health screening in the community; identify priority risk domains; select appropriate measures for screening identified risks; and establish a screening and referral protocol. In the course of carrying out these tasks with community partners, a Connections advisory board emerged and was formalized.

\section{Mapping eligibility requirements across agencies}

Solidifying a strong support system for use of Connections and acceptance of referrals was essential to the implementation 
of the screening and referral process. Providing appropriate referrals requires that referring agency staff and families understand basic eligibility requirements of agencies to which families are referred or to which they self-refer. To improve knowledge about eligibility requirements and to promote generation of appropriate referral across all partnering agencies, eligibility requirements were mapped out and provided to each agency, and, when relevant, are provided to families who receive Connections services.

\section{Identifying indicator and risk domains}

Identification of indicator and risk domains involved a 3-step process that included a review of the literature on child, parent, and family risks associated with significant infant mental health concerns, presentation of literature results to community partners, and community partner review and rating of indicator domains regarding severity of threat to social-emotional health of very young children in the community. In addition to the domain of child social-emotional delay or problem, 5 core indicator domains were selected on the basis of community partner review and ratings of indicator domains presented from the literature. These included the following: inappropriate and harsh parenting beliefs (Whiteside-Mansell, Bradley, Owens, Randolph, \& Cauce, 2003), parent depression, parent substance abuse, domestic partner abuse (Arellano, 1996; Windham et al., 2004; Wu et al., 2005), and any identified child developmental delay. In addition to the core indicators, 6 additional risk domains were identified, which included family history of child protection involvement (Hammond, 2003), lack of parent selfsufficiency, low maternal education (Aber, Jones, \& Cohen, 2000), maternal or child health concern, and teen parent status (Rothberg \& Weissman, 2002).

\section{Selecting measures}

Screening involved a multimethod, multisource approach, using measures that received highest ratings on both efficiency and technical soundness. Inclusion criteria, which reflected psychometric as well as practical considerations, were established for selecting screening measures. Consideration for inclusion required evidence of technical soundness based on published reports of adequate reliability and validity, sensitivity for detecting concerns among culturally diverse populations including women and adolescents, and established norms or empirically established cutoff scores for risk identification. Practical considerations included feasibility factors such as brevity, low cost, and ease of administration and scoring. On the basis of recommendations for maximizing sensitivity and efficiency (see Gilliam et al., 2005), it was determined that screening of core indicator domains should be conducted systematically using a relevant measure that met each of the criteria described above (see Table 2 for selected screening measures by concern domain).

Indicator and risk domains were weighted on the basis of how highly the domain was rated as a proximal risk for infant mental health problems with highest scores given to the most proximal indicators. To expedite systematic referral, a risk template was then created to record individual infant mental health indicators (ie, the Infant Mental Health Indicator Family Screening Profile; see Appendix A). A positive screen for any given core indicator domain is shown through shading on the Family Screening Profile. Positive screens on core risk domains are obtained when the relevant screening measure score falls within the range for concern based on published norms or cutoff scores for the particular measure. In addition, a cumulative risk score is generated on the Family Screening Profile based on the sum of positive screens across weighted domains.

\section{Screening and referral protocol}

As with each previous aspect of Connection's development, the screening and referral protocol was established with input and feedback from community partners. The screening and referral process, through which 
Table 2. Selected measures for concern domains ${ }^{\mathrm{a}}$

\begin{tabular}{|lc|}
\hline Domain & Measure \\
\hline $\begin{array}{l}\text { Child development } \\
\text { Child social-emotional } \\
\text { development }\end{array}$ & Ages and Stages Questionnaires (Squires, Potter, \& Bricker, 1995) \\
Parenting beliefs and attitudes & Ages and Stages Questionnaire-SE (Squires, Bricker, Twombly, \& \\
& Yockelson, 2001) \\
Adult-Adolescent Parenting Inventory (AAPI-2; Bavolek and \\
Karent depression & Keene, 1999) \\
& CESD (Centers for Epidemiological Studies of Depression; Radloff, \\
Substance abuse & 1977) \\
& Brief Michigan Alcohol Screening Test (Pokorny, Miller, \& Kaplan, \\
& 1972) \\
CRAFFT: A Brief Screening Test for Adolescent Substance Abuse \\
(Children's Hospital Boston, 2001) \\
\end{tabular}

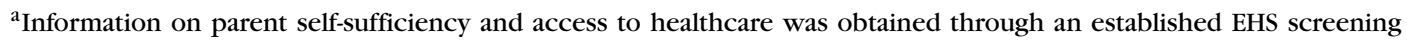
interview.

families progress from point of entry into Connections to completion, is described here and presented visually in Appendix B. The screening and referral process is initiated by phone call or in person within 1 week of the time that a family initially requests services for EHS or directly requests resource and referral support from Connections, which occurs when parents self-refer or are referred by other agencies. The screening and referral process is conducted in the family home if needed and typically requires a 2-hour visit and a follow-up phone call. This visit focuses on forging a partnership with parents to engage in systematic screening to determine resource needs and to develop an individualized referral plan for families to access services to meet those needs. This partnership is carried out through screening activities including structured interview, completion of a family wish list, and formal screening of infant mental health indicators. The screening portion of the visit requires approximately $1 \frac{1}{4}$ hours for completion.

The screening data, as recorded on the Family Screening Profile, are used to make referral decisions. Before providing referrals, parents and staff jointly complete a form that summarizes the family's current access to, and satisfaction with, services to address needs based on identified concern domains (eg, concern about possible child developmental delay, maternal depression, etc). Screening results are shared with parents and an individualized problem-solving approach is applied to the referral process. Referrals are provided to a variety of community agencies based on positive screens. Families with elevated cumulative risk scores on the Family Screening Profile are given priority enrollment status on the EHS wait list. Referral information is provided in a manner to help increase consumer knowledge about how to navigate and gain access to resources and services by using information-sharing permission and interagency agreements to help expedite referrals. This includes detailed verbal and written information about where to call or go, who to talk with and when, what to say, and what to expect. Additional referral supports are offered (eg, placing a call, with parent permission, to the local Part $\mathrm{C}$ program to request follow-up, providing a follow-up call to the parent for additional problemsolving and support to address access barriers).

\section{Securing an advisory board}

As a result of the initial collaboration efforts of community partners, a core group of 
agencies was recruited for the Connections advisory board (see Table 1). The board convenes 3 times a year and is a venue through which Connections shares information, engages in discussion, and receives input and guidance from community stakeholders. In addition to ongoing communication with the advisory board, the Connections staff provide progress reports to the community at large during monthly CCWC meetings for the purpose of informing larger community planning efforts.

\section{Timely and appropriate referral}

Timely and appropriate referral depends on timely entry into screening, rapid completion of the entire screening and referral process, and development of an individualized referral plan. While the creation of an efficient and effective screening protocol, in conjunction with the development of a brief, rapid mechanism for completing the screening and referral process is described above, a fundamental challenge remains. That is, regardless of efficiency, screening, and referral systems that fail to engage high-risk families are of little value. Therefore, considerable effort was given to identifying and cultivating service and staff characteristics important for engaging families facing multiple risks and stressors such as transportation needs, transient living situations, or language barriers. Among the proactive strategies employed to engage multiple risk families were the following: (1) meeting families in their homes, (2) conducting drop in visits, (3) rapid rapport-building with parents through use of a wish list that encourages parent disclosure about their own hopes and dreams for their child as well as family needs, (4) enhancing family motivation to participate by using the wish list as the joint purpose for conducting screening and referral, (5) delivering Connections services in the family's primary language, and (6) employing staff members who are well-educated, well-trained in cultural sensitivity and effective communication skills, as well as highly knowledgeable of existing community services.

\section{System evaluation component}

Baseline information regarding the family's initial access to, and satisfaction with, services is collected at the end of the first visit. Approximately 1 month following the initial visit, a second office or home visit occurs to conduct a follow-up interview about services parents are receiving, as well as their satisfaction with those services, and to gather anonymously reported information from parents about their satisfaction with the screening and referral process.

\section{Initial indications of effectiveness}

Between March 2003, when Connections began direct services, and May 2005, 283 families (including $92 \%$ of those entering the EHS wait list), who would otherwise not have been screened or who would have received highly fragmented screening, participated in comprehensive screening of infant mentalhealth indicators. The mean time from wait list entry to completion of screening and referral was 33 days. Comprehensive screening resulted in 1677 community referrals, based on individualized child and family needs to address concerns spanning the domains of early childhood intervention, maternal depression, domestic partner abuse, substance abuse, self-sufficiency, and early education and family support. With prioritization of EHS entry based on family risk scores, for families at highest risk (ie, in the top third of risk scores), wait time was cut in half. The mean wait for entry for families with maximum risk scores was 6 weeks. These families are at greatest risk not only for poor child outcomes (Aber et al., 2000; Samaan, 2000), but they have also been at extraordinary risk for program disengagement while waiting for services (Summers, Templeton-McMann, \& Fuger, 1997). EHS enrollment provides continued opportunities for monitoring as well as case management support for families to access multiple, complex community services systems. Opportunities for continued monitoring have been identified as a critical need for better serving children with or at risk for developmental 
delays and their families (Guralnick, 2005). On the basis of positive screens for developmental concerns, 1 out of 5 children was referred immediately for Part-C services. Overall family satisfaction ratings were high with $94 \%$ of families providing anonymous reports that the overall screening and referral process was helpful or very helpful.

\section{Program growtb}

In addition to indications of effectiveness described above, several examples of program growth have also occurred, which include the following: (1) growth in the number of agencies entering into an official Connection's partnership. By the 2005 fiscal year, 17 interagency agreements were established and more than 10 additional agencies continue to collaborate with the project at some level; (2) creation and use of an Access database system to provide increased efficiency and functionality of the data collected, not only for the current purposes of efficient referral decisions and analysis of community indicators of infant mental health, but also for future interagency use to expedite access to services as permitted by appropriate family releases and dictated by Health Insurance Portability and Accountability Act (HIPAA) compliance regulations; (3) expansion to accept community referrals for any family with a child younger than 4 years, rather than the initial requirement of entering the EHS waitlist; and (4) expansion of administration of developmental screening, including social-emotional development, to include all children younger than 4 years within a particular family rather than restricting administration to a target child only.

\section{CONCLUSION}

While communities face significant challenges to developing efficient and effective screening and referral systems, the economic and human costs of failing to assist families in making connections to receive needed services, especially at a time when families are motivated and seeking such services, is extraordinary. This report describes an EHS screening and referral system with preliminary indications of effectiveness, warranting further evaluation. Key program features (eg, a systematic approach to comprehensive screening of infant mental health risks for all families at the time they seek EHS services and immediate referral to a full array of appropriate services) were cultivated to counter common barriers limiting effectiveness (eg, narrow, nonsystematic screening often characterized by poor community collaboration and significant delay in screening).

Next steps include an analysis of infant mental health indicators at the community level, changes in parent access to, and satisfaction with, community services to address individual needs, and parent satisfaction with the Connections screening and referral process. These data will contribute to understanding of screening efficiency, identification of the most successful aspects of Connections and dimensions in need of refinement, and gaps between community services that families most want and need for promoting infant mental health and existing community services. This information along with continued collaborative feedback will be used to further advance capacity for interagency collaboration. Future plans include broader interagency staff particularly in the area of health, development of Web-based interagency resource information, and Web-based interagency access to the Connections database contingent on informed parent consent for information sharing with attention to HIPAA regulations.

\section{REFERENCES}

Aber, J. L., Jones, S., \& Cohen, J. (2000). The impact of poverty on the mental health and development of very young children. In C. Zeanah (Ed.), Handbook of infant mental bealth (pp. 113-128). New York: Guilford Press.

Appleyard, K., Egeland, B., van Dulmen, M. H., \& Sroufe, 
L. A. (2005). When more is not better: The role of cumulative risk in child behavior outcomes. Journal of Child Psychology and Psychiatry, 46(3), 235-245.

Arellano, C. M. (1996). Child maltreatment and substance abuse: A review of the literature. Substance Use and Misuse, 31(7), 927-935.

Bradley, R. H., Whitehead, L., \& Mundfrom, D. J. (1994). Early indications of resilience and their relation to experiences in the home environment of low birthweight, premature infants living in poverty. Child Development, 19, 301-318.

Bricker, D., Davis, M. S., Squires, J. (November 2001). Mental Health Screening in Young Children. Paper presented at the Early Intervention Conference, Hamilton, New Zealand.

Children's Hospital Boston. (2001). The CRAFFT: A brief screening test for adolescent substance abuse. Boston, MA: Children's Hospital Boston.

Gilliam, W. S., Meisels, S., \& Mayes, L. (2005). Screening and surveillance in early intervention systems. In $\mathrm{M}$. J. Guralnick (Ed.), The developmental systems approach to early intervention (pp. 73-98). Baltimore: Paul H. Brookes Publishing.

Glascoe, F. P., Foster, M., \& Wolraich, M. L. (1997). An economic analysis of developmental detection methods. Pediatrics, 99, 830-837.

Guralnick, M. J. (2005). An overview of the developmental systems model for early intervention. In M. J. Guralnick (Ed.), The developmental systems approach to early intervention (pp. 3-28). Baltimore: Brookes.

Hammond, R. (2003). Public health and child maltreatment prevention: The role of the centers for disease control and prevention. Child Maltreatment, 8, 8183.

Harbin, G. L. (2005). Designing an integrated point of access in the early intervention system. In M. J. Guralnick (Ed.), The developmental systems approach to early intervention (pp. 99-131). Baltimore: Brookes.

Harbin, G. L., Pelosi, J., Kameny, R., McWilliams, R., Kitsul, Y., Fox, E., et al. (2004). Identifying and predicting successful outcomes of coordinated service delivery. Chapel Hill: The University of North Carolina at Chapel Hill, NCL FPG Child Development Institute.

Kansas Action for Children, Inc. (2003, March). Invest Early: Good beginnings last a lifetime Kansas kids count data book. Topeka, KS: Author.

Mrazek, P., Biglan, A., \& Hawekins, J. D. (2004). Community monitoring systems: Tracking and improving the well-being of America's children and adolescents. Falls Church, VA: Society for Prevention Research.

Partnership for Children. (2004). 2004 report card and data briefing book: The status of Greater Kansas City's children and youth. Kansas City, MO: Author.

Pokorny, A. D., Miller, B. A., \& Kaplan, H. B. (1972) The brief MAST: A shortened version of the Michigan alcoholism screening test. American Journal of Psychiatry 129, 342-345.

Rothberg, A. W., \& Weissman, A. (2002). The development of programs for pregnant and parenting teens. Social Work in Health Care, 35, 65-83.

Samaan, R. A. (2000). The influence of race, ethnicity, and poverty on the mental health of children. Journal of Health Care for the Poor and Underserved, 1, 100110.

Sanders, M. R., Cann, W., \& Markie-Dadds, C. (2003). Why a universal population-level approach to the prevention of child abuse is essential. Child Abuse Review, 12, 145-154.

Squires, J., \& Bricker, D. (2007). An activity based approach to developing young children's socialemotional competence. Baltimore: Paul H. Brookes.

Squires, J., Bricker, D., Twombly, F., \& Yockelson, M. S. (2001). Ages and stages questionnaire: Social emotional (ASQ: SE): A parent completed child monitoring system. Baltimore: Paul $\mathrm{H}$. Brookes Publishing.

Squires, J., Potter, L., \& Bricker, D. (1995). Ages and stages questionnaire: A parent-completed child monitoring system. Baltimore: Paul H. Brookes Publishing.

Summers, J. A., Templeton-McMann, O., \& Fuger, K. (1997). Critical thinking: A method to identify best practices in serving families with multiple challenges. Topics in Early Childhood Special Education, 17, 27-52.

U.S. Census Bureau. (2000). U.S. Census 2000 results. Prepared by the Unified Government of Wyandotte County, Kansas City, KS.

Wu, S. S., Ma, C. X., Carter, R. L., Ariet, M., Feaver, E. A., Resnick, M. B., \& Roth, J. (2005). Risk factors for infant maltreatment: A population-based study. Child Abuse and Neglect, 28, 1253-1264.

Windham, A. M., Rosenberg, L., Fuddy, L., McFarlane, E., Sia, C., \& Duggan, A. K. (2004). Risk of motherreported child abuse in the first 3 years of life. Child Abuse and Neglect, 28, 645-667.

Whiteside-Mansell, L., Bradley, H., Owens, M. T., Randolph, S. M., \& Cauce, A. M. (2003). Parenting and children's behavior at 36 months: Equivalence between African American and European American mother-child dyads. Parenting, 3, 197-234.

Zeanah, C. H., \& Zeanah, P. D. (2000, October). Towards a definition of infant mental bealth. Paper Presented at the Early Head Start and Migrant Head Start Infant Mental Health Forum, Administration on Children, Youth, and Families, Washington, DC. 


\section{Appendix A}

\section{Infant Mental Health Indicators \\ Family Screening Profile}

Brief Screener Index

\begin{tabular}{lllllll}
\hline $\begin{array}{c}\text { Index } \\
\text { Score }\end{array}$ & $\mathbf{4}$ & $\mathbf{3}$ & $\mathbf{3}$ & $\mathbf{1}$ & $\mathbf{1}$ & $\mathbf{1}$ \\
\hline Domain & $\begin{array}{c}\text { Child social } \\
\text { emotional } \\
\text { delay/ } \\
\text { concern }\end{array}$ & $\begin{array}{c}\text { Parent } \\
\text { depression }\end{array}$ & $\begin{array}{c}\text { Risky } \\
\text { parenting } \\
\text { beliefs }\end{array}$ & $\begin{array}{c}\text { Parent } \\
\text { substance } \\
\text { abuse }\end{array}$ & $\begin{array}{c}\text { Other child } \\
\text { development } \\
\text { delay }\end{array}$ & $\begin{array}{c}\text { Domestic } \\
\text { partner } \\
\text { abuse }\end{array}$ \\
& & & & & \\
\hline
\end{tabular}

${ }^{a}$ Identified concerns are shaded

Interview Index

\begin{tabular}{|c|c|c|c|c|c|c|c|c|}
\hline $\begin{array}{l}\text { Index } \\
\text { Score }\end{array}$ & 3 & 1 & 2 & 3 & 1 & 1 & 1 & 1 \\
\hline Domain & $\begin{array}{l}\text { History of } \\
\text { child } \\
\text { protec- } \\
\text { tion } \\
\text { involve- } \\
\text { ment }\end{array}$ & \multicolumn{3}{|c|}{$\begin{array}{l}\text { Self-sufficiency } \\
\text { concern }(1= \\
\text { federal poverty; } \\
2=\text { unable to } \\
\text { purchase food, } \\
\text { heating/cooling } \\
\text { utilities, medicine } \\
3=\text { homeless) }\end{array}$} & $\begin{array}{l}\text { Maternal } \\
\text { low Ed/ } \\
\text { special } \\
\text { Ed }\end{array}$ & $\begin{array}{c}\text { Maternal } \\
\text { health } \\
\text { concern }\end{array}$ & $\begin{array}{c}\text { Teen } \\
\text { parent } \\
\text { status }\end{array}$ & $\begin{array}{l}\text { Child } \\
\text { health } \\
\text { concern }\end{array}$ \\
\hline
\end{tabular}

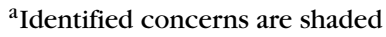

Index Scores

Brief screener results index 6

Interview results index 3

Infant mental health risk index 9

Note: Families with Infant Mental Health Risk Index scores $\geq 4$ receive priority EHS enrollment status in addition to immediate referral support to access other needed community services. 


\section{Appendix B}

\section{Connections Protocol: Family Entry to Completion}

All women who are pregnant or parents who have a child under 1 year of age who request Early Head Start, Healthy Start, or Teen Parenting support through Project EAGLE Community Programs are invited to participate in the Connections screening and referral process.

In addition, any family with a child younger than 4 years of age living in the county and who is referred to Connections by a community partner is contacted within the first week of referral to schedule an initial Connections visit.

\section{Home or office screening and referral visit}

Within 1 week of initial contact, a 2-hour home or office visit is conducted with each family who has entered Connections.

\section{Screening components}

Interview

Wish list

Screening measures

Baseline measure service needs/satisfaction

\section{Referral}

Individualized family plan

(verbal and written)

\section{Possible referral outcomes}

\section{For all positive screens}

- Direct referral is provided for any relevant community agency based on positive screen or direct parent request.

- Problem-solving support is provided to promote service access.

\section{For all families who meet criteria for priority EHS status}

In addition to individual agency referrals, a family is given priority for EHS enrollment to expedite case management supports and ongoing monitoring.

\section{Follow-up phone call}

Referral follow-up support

Problem-solving for referral follow-through

\section{Exit survey}

Family needs and services assessment

Parent satisfaction survey

Current articles- http://depts.washington.edu/isei/iyc/iyc_comments.html

To make a comment or to ask questions about this article:

http://admin.chdd.washington.edu/isei/review/commentbox_baggett.htm 